\title{
Prevalence and Genetic Characterizations of Enterocytozoon Bieneusi in Captive Red Pandas (Ailurus Fulgens) In Sichuan Province, Southwestern China
}

\section{Yang-yang Zeng}

Sichuan Agricultural University https://orcid.org/0000-0002-1496-6055

\section{Wan-Yu Meng}

Sichuan Agricultural University

\section{Song-Rui Liu}

Sichuan Key Laboratory of Conservation Biology for Endangered wildlife.

Jin-Chuan Yao

Sichuan Agricultural University

\section{Ming He}

China Consercation and Research Center for Giant Panda

\section{Li-Qin Wang}

Chengdu Zoo

\section{Zhi-Li Zuo}

Chengdu Zoo

\section{Chan-Juan Yue}

Sichuan Key Laboritory of Conservation Biology for Endangered wildlife.

\section{Lei Deng}

Sichuan Agricultural University

\section{Guangneng Peng ( $\nabla$ pgn.sicau@163.com )}

Sichuan Agricultural University https://orcid.org/0000-0002-9898-5754

\section{Research Article}

Keywords: Microsporidia, Enterocytozoon bieneusi, Red panda, Ailurus fulgens,

Posted Date: August 2nd, 2021

DOl: https://doi.org/10.21203/rs.3.rs-733215/v1

License: (c) (1) This work is licensed under a Creative Commons Attribution 4.0 International License. Read Full License 
Page $2 / 18$ 


\section{Abstract}

Background: Enterocytozoon bieneusi (E. bieneusi) can infect a broad range of animals, and also the major pathogen for human microsporidiosis. The risk of zoonosis is uncertain because of limited research on red pandas. In addition, the semi-free range breeding enables the red panda direct contact with tourists. It is essential to investigate the prevalence and genotypes and to evaluate the safety of this breeding mode.

Methods: Based on nested PCR, 198 fecal specimens were sampled from 6 zoos in Sichuan province from July 2020 to December 2020, to identify positive samples by amplifying the internal transcribed spacer (ITS) region of ribosomal RNA with specific primers. The correlation analysis of infection rate was carried out between different breeding modes (captive and semi-free-range). To cluster the identified genotypes with related genotypes to deduce zoonotically potential by phylogenetic analysis. In addition, Multilocus genotypes (MLGs) in ITS-positive samples were performed using the Multilocus Sequence Typing (MLST) tool.

Results: The Polymerase Chain Reaction (PCR) results showed that $12.1 \%(24 / 198)$ samples were positive for E.bieneusi. The infection rates varied from $0 \%$ to $18.0 \%$ in different zoos and were significantly different in different breeding methods $\left(\chi^{2}=5.442, P=0.0197\right)$. Genotypes $D, S C 02$, and SCR1(novel) were clustered in zoonotic group 1, while genotype PL2 is clustered in group 2-like with uncertain risk by phylogenetic analysis. Furthermore, 3 distinct multilocus genotyping were formed in ITSpositive isolates.

Conclusions: These results revealed the circulating of E. bieneusi in zoo red pandas, indicating that red pandas may be a source of human microsporidiosis and that semi-free range breeding mode as a risk factor increased the $E$. bieneusi infection rate and potential cross-species transmission.

\section{Background}

Microsporidia is a group of opportunistic obligated intracellular parasites, which are currently classified as fungi and their host range is very wide, including most invertebrates, vertebrates, and humans [1-3]. At least 1500 microsporidia species, which belong to 200 genera, have been formally described till now, 17 of which were reported to infect humans $[4,5]$. E. bieneusi is one of the most common pathogens of human microsporidiosis, accounting for $90 \%$ of human cases of the disease [6, 7]. E. bieneusi infection can cause acute to chronic diarrhea, malabsorption, inflammation of the respiratory tract, and acalculous cholecystitis in people, and even deadly severe diarrhea in people with immunosuppression [8-10].

Through sequencing analysis of the ITS region of the ribosomal RNA (rRNA), more than 500 genotypes were identified and divided into 11 groups based on cluster analysis [11]. Group 1, including most species, was mainly comprised of genotypes with a wide host range and have higher zoonotic potential, including more than 40 genotypes identified in both humans and animals [12]. Group 2, the secondlargest, was previously thought to only infect cattle, but with the discovery of a wider host range for some 
genotypes in this group, suggesting potential public health issues to an extent [5]. The majority of the genotypes from groups 3-11 appear to be more host-specific, and therefore, lead to a slight or unknown threat to public health [5]. However, the single ITS loci cannot differentiate E. bieneusi isolates that are genetically closely related [13]. A higher resolution tool, MLST, was developed and used for sub genotyping [14]. To date, MLST tools are already used in more than 167 ITS genotypes to study the genetic characters by distinguishing the repeat sequence and single nucleotide polymorphisms (SNPs) $[14,15]$.

In recent years, the zoonotic genotypes of E. bieneusi have been found in many animal groups, including birds, non-human primates, domestic and captive wild animals [16-27]. Past studies have shown Genotypes D and EbpC occurred in Chinese red pandas $[13,19,28]$. At the same time, these two genotypes have been frequently reported in the Chinese population as well, suggesting the risk of red pandas as a potential reservoir of zoonotic pathogens.[2, 29]. The captive populations of red pandas in China are mostly concentrated in zoos in southwest China, especially in Sichuan. However, no regional large-scale epidemiological investigation has been carried out yet in these regions. Furthermore, some zoos breed red pandas in semi-free-range mode, the safety for both humans and animals is uncertain; the zoonotic potential in different regions and breeding modes should be assessed. Therefore, we carried out this study.

\section{Methods}

\section{Sample collection}

We collected 198 fresh fecal specimens from 6 zoos or breeding sites of 5 regions in Sichuan province, between July 2020 and December 2020 (Fig. 1, Table 2). Most animals are kept with a barbed wire fence nearly 1 meter high separating the animals from visitors. We divided the animals into two groups depending on the range of movement and the level of human connection. Group i) captive red panda groups which live alone and do not interact with each other, only contact with keepers; Group ii) semi-freerange, which means red panda groups can travel freely within certain limits and may interact with other groups or visitors. All samples were collected by sterile gloves within 24 hours after defecation and immediately transported in a cold box to the clinic veterinary laboratory of Sichuan Agricultural University, then stored at $-20^{\circ} \mathrm{C}$ till DNA extraction.

\section{DNA extraction and PCR amplification}

All samples were cryogenically centrifuged at $3000 \mathrm{rpm}$ for $5 \mathrm{~min}$ after washing and filtering out impurities. Extracting genome DNA from $200 \mathrm{mg}$ pretreated feces using E.Z.N.A.® stool DNA Kit (OMEGA, Biotek Inc. USA) following the recommended procedures. Add $200 \mu$ l kit Solution Buffer into each extracted DNA sample and stored at $-20^{\circ}$ until use.

The positive samples were screened by an approximately $390 \mathrm{bp}$ amplified fragment in the ITS region

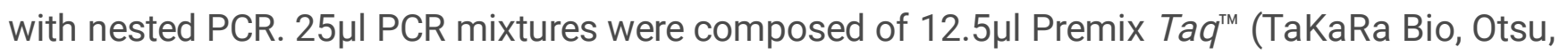


Japan),8.5 $\mu \mathrm{lddT} \mathrm{H}_{2} \mathrm{O}, 2 \mu \mathrm{l}$ primers and $2 \mu \mathrm{l}$ genomic DNA. To increase the detection of positive isolates, use two pairs of different ITS primers (ITS1, ITS2) to amplify the specimens. The primers and reaction temperatures of PCR amplification were referenced from previous articles [14, 30, 31](Table 2). All PCR reactions arranged positive and negative controls. Secondary PCR products were stained by GoldView ${ }^{\text {TM }}$ and visualized in $1.0 \%$ ethidium bromide-stained agarose gel by electrophoresis. To further identify subgenotype characteristics, positive ITS specimens were amplified by MLST in loci MS1, MS3, MS4, and MS7[14].

\section{Sequencing and phylogenetic analysis}

To ensure accuracy, the nucleotide sequences of positive specimens were sent to TsingKe Biological Technology (Chengdu, China) for bi-directional sequencing. Using Basic Local Alignment Search Tool (BLAST) (http://www.ncbi.nlm.ni-h.gov/BLAST/) to identify the genotypes of E. bieneusi. Then Aligned the obtained sequences and the reference sequences from GenBank with Clustal X 1.83 (http://www.clustal.org/). The phylogenetic trees were reconstructed by the MEGA 7.0 program (version 7.0.26), specifically choosing the neighbor-joining method, Kimura 2-parameter model, and 1000 bootstrap replicates. The genotype names followed previously published ones if the obtained sequences are identical to known genotypes. Novel genotypes were identified according to the single nucleotide substitutions, deletions, or insertions in the $243 \mathrm{bp}$ region of the ITS gene and named by the nomenclature rules of the previous study [32].

Statistics analyses.

The chi-square test and exact probability test were used for significant differences of $E$. bieneusi infection among different regions and different groups based on SPSS 26.0 software (https://spss.en.softonic.com/). Set 95\% confidence intervals (Cls) and odds ratios (ORs)dang. When P value $\leq 0.05$, the differences in results were considered significant.

Nucleotide sequence GenBank accession numbers All ITS and MLST sequences obtained in this study were submitted to the GenBank database. Acquired the accession number MW880217-MW880236, MW880238-MW880241, and MW922590-MW922622.

\section{Results}

\section{Occurrence and genotype of E. bieneusi in red pandas}

Among 198 fecal samples detected by nested PCR, 24 tested positives for E. bieneusi, the overall infection rate reached $12.1 \%(24 / 198)$ (Table 1). The infection rates of E. bieneusi were $18.0 \%(18 / 100)$, 7.1\% (3/42), 7.1\% (1/14), 9.1\% (1/11),0\% (0/18),7.7\% (1/13) in Chengdu Research Base of Giant Panda Breeding(CDBGPB), Sanlang Resort(SLR), Bifengxia Zoo(BFXZ), Chengdu Zoo(CDZ), Wolong China Giant Panda Garden(WCGPG), Panda Valley(PV), respectively. The differences among different regions were not significant $\left(X^{2}=7.364, d f=5, P=0.1949>0.05\right)$. In groups, Group i was $5.9 \%(5 / 85)$, and Group ii 
reached up to $16.8 \%(19 / 113)$. There was a significant difference between in different groups $\left(\chi^{2}=5.442\right.$, $\mathrm{df}=1, \mathrm{P}=0.0197<0.05)$.

Table 1

Sampling information and epidemic results of E. bieneusi in red pandas in this study.

\begin{tabular}{|c|c|c|c|c|c|c|}
\hline Group & Region & Site & $\begin{array}{l}\text { Breeding } \\
\text { mode }\end{array}$ & $\begin{array}{l}\text { No. of } \\
\text { samples }\end{array}$ & $\begin{array}{l}\text { No. of positive } \\
(\%)\end{array}$ & $\begin{array}{l}\text { ITS } \\
\text { genotypes }\end{array}$ \\
\hline \multirow[t]{4}{*}{ प } & Chenghua & $\mathrm{CDZ}$ & Captive & 11 & $1(9.1 \%)$ & D \\
\hline & Wenchuan & WLCGPG & Captive & 18 & $0(0.0 \%)$ & \\
\hline & Chongzhou & SLR & Captive & 42 & $3(7.1 \%)$ & SCR1 \\
\hline & Ya'an & BFXZ & Captive & 14 & $1(7.1 \%)$ & D \\
\hline \multirow[t]{2}{*}{ ( } & Chenghua & CDRBGPB & $\begin{array}{l}\text { Semi-free- } \\
\text { range }\end{array}$ & 100 & 18 (18.0\%) & PL2 \\
\hline & Dujangyan & PV & $\begin{array}{l}\text { Semi-free- } \\
\text { range }\end{array}$ & 13 & $1(7.7 \%)$ & SCO2 \\
\hline $\begin{array}{l}\text { Note: C } \\
\text { Bifeng> }\end{array}$ & $\begin{array}{l}\text { GPB: Chenc } \\
\text { Zoo; CDZ: C }\end{array}$ & $\begin{array}{l}\text { Research E } \\
\text { igdu Zoo; I }\end{array}$ & $\begin{array}{l}\text { se of Giant } \\
\text { SGPG: Wolo }\end{array}$ & $\begin{array}{l}\text { Breedir } \\
\text { ha Gian }\end{array}$ & $\begin{array}{l}\text { Sanlang Resort; } \\
\text { Garden; PV: Par }\end{array}$ & XZ: \\
\hline
\end{tabular}

All 24 positive isolates were successfully sequencing. observed Four genotypes, including 3 described genotypes (D, PL2, and SC02) and one novel genotype SCR1. The obtained ITS gene sequences of genotypes D, PL2, and SC02 were identical with reference sequences KY950534, MT497891, and KU852476 from GenBank, respectively. The homology was $98.6 \%$ between novel genotype SCR1 and genotype FJL (MK357781), with one single nucleotide substitution and one insertion. Among them, genotype PL2 $(n=18)$ was dominant and genotype SCR1 $(n=3)$ was ranked second. Both SC02 and D only identified one sample. 
Table 2

The primers and annealing temperatures of PCR amplification.

\begin{tabular}{|c|c|c|c|c|}
\hline Gene & Primer sequences $\left(5^{\prime}-3^{\prime}\right)$ & AT $\left({ }^{\circ} \mathrm{C}\right)$ & Size (bp) & Reference \\
\hline \multirow[t]{4}{*}{ ITS1 } & F1: GATGGTCATAGGGATGAAGAGCTT & \multirow[t]{2}{*}{55} & \multirow[t]{4}{*}{392} & [31] \\
\hline & R1: AATACAGGATCACTTGGATCCGT & & & \\
\hline & F2: AGGGATGAAGAGCTTCGGCTCTG & \multirow[t]{2}{*}{55} & & \\
\hline & R2: AATATCCCTAATACAGGATCACT & & & \\
\hline \multirow[t]{4}{*}{ ITS2 } & F1: GGTCATAGGGATGAAGAG & 57 & 390 & [14] \\
\hline & R1: TTCGAGTTCTTTCGCGCTC & & & \\
\hline & F2: GCTCTGAATATCTATGGCT & 55 & & \\
\hline & R2: ATCGCCGACGGATCCAAGTG & & & \\
\hline \multirow[t]{4}{*}{ MS1 } & F1: AAGTTGCAAGTTCAGTGTTTGAA & \multirow[t]{2}{*}{58} & \multirow[t]{4}{*}{676} & [14] \\
\hline & R1: GATGAATATGCATCCATTGATGTT & & & \\
\hline & F2: TTGTAAATCGACCAAATGTGCTAT & \multirow[t]{2}{*}{58} & & \\
\hline & R2: ACATAAACCACTAATTAATGTAAC & & & \\
\hline \multirow[t]{4}{*}{ MS3 } & F1: CAAGCACTGTGGTTACTGTT & \multirow[t]{2}{*}{55} & \multirow[t]{4}{*}{537} & [14] \\
\hline & R1:AAGTTA GGGCATTTAATAAAATTA & & & \\
\hline & F2: GTTCAAGTAATTGATACCAGTCT & \multirow[t]{2}{*}{55} & & \\
\hline & R2: CTCATTGAATCTAAATGTGTATAA & & & \\
\hline \multirow[t]{4}{*}{ MS4 } & F1: GCATATCGTCTCATAGGAACA & \multirow[t]{2}{*}{55} & \multirow[t]{4}{*}{885} & [30] \\
\hline & R1: GTTCATGGTTATTAATTCCAGAA & & & \\
\hline & F2: CGA AGTGTACTACATGTCTCT & \multirow[t]{2}{*}{55} & & \\
\hline & R2: GGACTTTAATAAGTTACCTATAGT & & & \\
\hline \multirow[t]{4}{*}{ MS7 } & F1: GTTGATCGTCCAGATGGAATT & \multirow[t]{2}{*}{55} & \multirow[t]{4}{*}{471} & [14] \\
\hline & R1: GACTATCAGTATTACTGATTATAT & & & \\
\hline & F2: CAATAGTAAAGGAAGATGGTCA & \multirow[t]{2}{*}{55} & & \\
\hline & R2: CGTCGCTTTGTTTCATAATCTT & & & \\
\hline $\begin{array}{l}\text { Note } \\
\text { prim }\end{array}$ & $\begin{array}{l}\text { nnealing temperatures; F1:Forward pri } \\
\text { R; F2:Forward primer of the second PC }\end{array}$ & ary PC & Reverse & of the \\
\hline
\end{tabular}


Phylogenetic analysis results (Fig. 2) showed that genotype D and SCR1 belonged to subgroup1a in Group 1. SC02 is clustered in subgroup 1b of Group 1, and PL2 formed a separate clade related to Group 2 [33]. All genotypes were human-pathogenic [31]. To further analyze the gene polymorphism and the sub-genotype by using the MLST (Table 3). The amplification efficiencies in loci MS1, MS3, MS4, and MS7 were $25.0 \%(6 / 24), 45.8 \%(11 / 24), 16.7 \%(4 / 24)$, and 50.0\%(12/24), respectively. Only 3 genotype PL2 specimens tested positive in these five loci. Correspondingly generated 1, 2, 4, and 1 types in MS1, MS3, MS4, and MS7 locus based on different nucleotide repeats and SNPs. 3 distinct MLGs were formed in genotype PL2, and some degree of sequence polymorphism was shown. 
Table 3

Multilocus genotypes of positive E.bieneusi isolates from red pandas in this study.

\begin{tabular}{|c|c|c|c|c|c|c|c|}
\hline \multirow{2}{*}{ Sampling sites } & \multirow{2}{*}{$\begin{array}{l}\text { Sampling } \\
\text { no. }\end{array}$} & \multirow[t]{2}{*}{ ITS genotype } & \multicolumn{5}{|c|}{ Multilocus genotypes } \\
\hline & & & MS1 & MS3 & MS4 & MS7 & MLGs \\
\hline \multirow[t]{18}{*}{ CDRBGPB } & 3 & PL2 & & & & & \\
\hline & 5 & PL2 & & type1 & & type1 & \\
\hline & 10 & PL2 & & type1 & & type1 & \\
\hline & 11 & PL2 & type1 & type1 & type1 & type1 & MLG1 \\
\hline & 12 & PL2 & type1 & type1 & & & \\
\hline & 15 & PL2 & & & & & \\
\hline & 16 & PL2 & & & & type1 & \\
\hline & 18 & PL2 & & & & & \\
\hline & 19 & PL2 & type1 & & type1 & type1 & \\
\hline & 21 & PL2 & & & & & \\
\hline & 22 & PL2 & & type1 & & & \\
\hline & 40 & PL2 & & & & & \\
\hline & 43 & PL2 & & & & & \\
\hline & 47 & PL2 & & type1 & & type1 & \\
\hline & 63 & PL2 & & type1 & & type1 & \\
\hline & 86 & PL2 & type1 & type1 & type2 & type1 & MLG2 \\
\hline & 93 & PL2 & type1 & & & type1 & \\
\hline & 95 & PL2 & type1 & type1 & type3 & type1 & MLG3 \\
\hline SLR & 122 & SCR1 & & & & & \\
\hline SLR & 136 & SCR1 & & type1 & & type1 & \\
\hline SLR & 145 & SCR1 & & & & & \\
\hline BFXZ & 174 & $\mathrm{D}$ & & type2 & & & \\
\hline PV & 185 & SC02 & & & & type1 & \\
\hline CDZ & 211 & D & & & & & \\
\hline
\end{tabular}


This study sampled 6 zoos in 5 different regions and demonstrated the prevalence and genotype characterization of E. bieneusi among captive red pandas in Sichuan province, southwest China. In this study, red pandas in Group ii have a significantly higher prevalence of infection than Group i. The difference may be related to the larger range of red pandas and the more frequent contact between different animal populations and between animals and humans under the semi-free-range breeding mode. Meanwhile, higher prevalence indicated that the safety of semi-free-range breeding mode needs to be carefully regulated, due to zoonotic transmission potential.

The overall prevalence of this study was $12.1 \%$, which is close to the previous report of red pandas in Shaanxi province, northwestern China, $11.1 \%-13.9 \%[13,28]$. In the present study, the prevalence of Chengdu Zoo and Bifengxia Zoo was 7.1\% (1/14) and $9.1 \%$ (1/11), both were lower than former prevalence data (10.6\% and 29.7\%)[19]. The differences in E. bieneusi prevalence between studies may be influenced by geographical region, sample size, sampling time, animal health condition, breeding mode, and population density[34-36]. The role of those factors can be further examined. As the closest phylogenetic relationship animal with red panda [37], the American raccoon (Procyon lotor), also possessed a higher prevalence $(27.3 \%, 15 / 55)[27,31]$. Red pandas have a low overall prevalence compared to other common zoo species with a sampling size of more than 20 isolates (Table 4). Ignoring the interference of other factors, the adaptation of E.bieneusi to the red panda may be in the middle level. 
Table 4

The prevalence of E.bieneusi in some species of Chinese zoos.

\begin{tabular}{|c|c|c|c|c|}
\hline Family & Species & $\begin{array}{l}\text { No. of } \\
\text { tested }\end{array}$ & $\begin{array}{l}\text { No.of } \\
\text { positive (\%) }\end{array}$ & Reference \\
\hline Ailuridae & Red panda (Ailurus fulgens) & 198 & $24(12.1 \%)$ & $\begin{array}{l}\text { This } \\
\text { study }\end{array}$ \\
\hline \multirow[t]{4}{*}{ Cercopithecidae } & $\begin{array}{l}\text { Golden snub-nosed monkey } \\
\text { (Cercopithecus kandti) }\end{array}$ & 160 & $74(46.2 \%)$ & [26] \\
\hline & Rhesus macaque (Macaca mulatta) & 304 & $60(19.7 \%)$ & {$[16,33]$} \\
\hline & $\begin{array}{l}\text { Hamadryas baboon (Papio } \\
\text { hamadryas) }\end{array}$ & 21 & $6(28.6 \%)$ & [42] \\
\hline & $\begin{array}{l}\text { Cynomolgus monkey (Macaca } \\
\text { fascicularis) }\end{array}$ & 80 & $47(58.8 \%)$ & {$[16,18]$} \\
\hline \multirow[t]{2}{*}{ Cebidae } & Squirrel monkey (Saimirisp.) & 43 & $17(39.5 \%)$ & [16] \\
\hline & Black-capped capuchin (Cebus apella) & 22 & $6(27.3 \%)$ & [42] \\
\hline \multirow[t]{2}{*}{ Ursidae } & Giant panda (Ailuropoda melanoleuca) & 200 & $69(34.5 \%)$ & [16] \\
\hline & Asiatic black bears (Ursus thibetanus) & 106 & $29(27.4 \%)$ & [42] \\
\hline Bovidae & $\begin{array}{l}\text { Golden takin (Budorcas taxicolor } \\
\text { bedfordi) }\end{array}$ & 191 & $28(14.7 \%)$ & [42] \\
\hline Anatidae & Whooper swans (Cygnus cygnus) & 467 & $35(7.5 \%)$ & [42] \\
\hline Moschidae & Musk deer (Moschus berezovskii) & 223 & $38(17.0 \%)$ & [42] \\
\hline Lemuridae & Ring-tailed lemur (Lemur catta) & 45 & $11(24.0 \%)$ & [42] \\
\hline Hominidae & $\begin{array}{l}\text { Bornean orangutan (Pongo } \\
\text { pygmaeus) }\end{array}$ & 23 & $4(17.4 \%)$ & [16] \\
\hline \multirow[t]{2}{*}{ Macropodidae } & Red kangaroo (Macropus rufus) & 38 & $14(36.8 \%)$ & [38] \\
\hline & Grey kangaroo (Macropus fuliginosu) & 23 & $0(0.0 \%)$ & [38] \\
\hline
\end{tabular}

Four observed genotypes in this study are completely different from genotypes previously identified in red panda (EBPC, CHB1) $[13,19,28]$. As the dominant genotype, PL2 was only found in masked palm civets (Paguma larvata) and formed a clade related to Group 2 in phylogenetic analysis. [33, 39]. All positive isolates from CDRBGPB were PL2, which revealed red panda is a new host of genotype PL2, which expands the host range of this genotype. The high prevalence in CDRBGPB indicates that red pandas may occur inter-species transmission and be an adaptive host of PL2, but the infection route is unknown and needs more future research. Long-term monitoring and further study are also required to ascertain whether the semi-free-range breeding mode has increased the transmission of E. bieneusi within the red panda populations and the potential risk of cross-specific transmission. Both Chengdu Zoo and Bifengxia Zoo observed only 1 genotype $D$ isolates. Considering that genotype $D$ has previously been discovered in 
the above-mentioned zoos, pathogens originated from other animal hosts are possible [26, 40]. Genotype SC02 was once detected in humans and various animals such as Asian black bears, Tibetan blue bears, sun bears, raccoons, horses, and squirrels $[19,41,42]$. The exchange and recombination in ITS genes of E.bieneusi may have resulted in genetic diversity and the new genotype SCR1 [43].

MLST analysis showed that amplification efficiency is different in various ITS genotypes, which is consistent with previous studies [11]. We found 3 MLGs, which showed higher genetic diversity in PB isolates than the other 5 sampling sites; however, the influence of sample size cannot be excluded. MLST tools revealed higher resolution and genetic diversity than ITS sequence genotyping, which is according to the conclusions of previous studies[18, 41, 42,44]. The amplification efficiency of locus MS4 was higher than MS1, MS4, and MS7, which was inconsistent with previous reports[14, 45].

\section{Conclusions}

In conclusion,this study clear up the E.bieneusi prevalence and genotypes of captive red pandas in Sichuan zoos. Resultantly, captive red pandas are new hosts for identified genotypes, and the semi-free range breeding mode can be a significant risk factor in E.bieneusi prevalence. Genotype D, SC02, and SCR1 phylogenetically clustered in Group 1 indicated the red panda with nonnegligible zoonotic risk and can be a potential source of human-pathogenic microsporidia. Zoos should continue to monitor the epidemic of red pandas E.bieneusi infection and take necessary measures to reduce the infection. The risk of zoonosis in different breeding modes also needs to be further studied to ensure the safety of animals and human beings.

\section{List Of Abbreviations}

ITS: Internal Transcribed Spacer; MLGs: Multilocus genotypes; MLST: Multilocus Sequence Typing; PCR: Polymerase chain reaction; rRNA: ribosomal RNA; BLAST: Basic Local Alignment Search Tool; AT:Annealing temperatures; $\mathrm{Cl}$ : Confidence Intervals; ORs: Odds Ratios; CDBGPB: Chengdu Research Base of Giant Panda Breeding; SLR: Sanlang Resort; BFXZ: Bifengxia Zoo; CDZ: Chengdu Zoo; WCGPG: Wolong China Giant Panda Garden; PV: Panda Valley;

\section{Declarations}

\section{Ethics approval and consent to participate}

This study Collect fecal samples of captive red pandas with the permission of the owner and/or administrator. All procedures are strictly followed by the requirements of the Procedures and Guidelines for Animal Ethics of the People's Republic of China.

\section{Consent for publication}

Not applicable. 
Availability of data and materials

The datasets collected and/or analyzed during the current study are available

from the corresponding author upon reasonable request.

\section{Competing interests}

The authors declare that they have no competing interests

\section{Funding}

This work was supported by the Chengdu Giant Panda Breeding Research Foundation (CPF2017-12).

\section{Authors' contributions}

Yang-Yang Zeng, Wan-Yu Meng, and Song-Rui Liu are equally contributed to this study.

\section{Competing interests}

All authors related in this study have no conflicts interests and competing interests.

\section{Acknowledgments}

We would like to thank all the staff in those zoos for helping collect samples and thank all the classmates and students for their help and assistant in experimenting. And special acknowledgment to the support of Chengdu Giant Panda Breeding Research Foundation.

\section{Authors' information}

Yang-Yang Zeng ${ }^{1+}:$ 345162804@qq.com; Wan-Yu Meng ${ }^{1+}:$ 1394138541@qq.com; Sonr-Rui Liu²t: 405536517@qq.com; Jin-Chuan Yao': 463435539@qq.com; Ming He³: 327636780@qq.com; Li-Qin Wang4: 523064928@qq.com; Zhi-Li Zuo4: 269394674@qq.com; Chan-Juan Yue 2*: 269394674@qq.com; Lei Deng ${ }^{1 *}:$ cd1931023@163.com; Guang-Neng Peng ${ }^{1 *}$ :pgn.sicau@163.com;

\section{References}

1. Keeling P. Five questions about microsporidia. PLoS Pathog. 2009;5:e1000489.

2. Wang SS, Wang RJ, Fan XC, et al. Prevalence and genotypes of Enterocytozoon bieneusi in China. Acta Trop. 2018;183:142-52.

3. Stentiford GD, Bass D, Williams B. Ultimate opportunists-The emergent Enterocytozoon group Microsporidia. PLoS Pathog. 2019;15:e1007668.

4. Vavra J, Lukes J. Microsporidia and 'the art of living together'. Adv Parasitol. 2013;82:253-319. 
5. Li W, Feng Y, Santin M. Host Specificity of Enterocytozoon bieneusi and Public Health Implications. Trends Parasitol. 2019;35:436-51.

6. Mathis A, Weber R, Deplazes P. Zoonotic potential of the microsporidia. Clin Microbiol Rev. 2005;18:423-45.

7. Matos O, Lobo ML, Xiao L. Epidemiology of Enterocytozoon bieneusi Infection in Humans. Journal of Parasitology Research. 2012;2012:1-19.

8. Didier ES, Weiss LM. Microsporidiosis. Current status. Curr Opin Infect Dis. 2006;19:485-92.

9. Singh I, Sheoran AS, Zhang Q, Carville A, Tzipori S. Sensitivity and specificity of a monoclonal antibody-based fluorescence assay for detecting Enterocytozoon bieneusi spores in feces of simian immunodeficiency virus-infected macaques. Clin Diagn Lab Immunol. 2005;12:1141-4.

10. Xin-Li Z, Huan-Huan Z, Gangxu R, et al. Genotyping and zoonotic potential of Enterocytozoon bieneusi in cattle farmed in Hainan Province, the southernmost region of China. Parasite (Paris). 2020;27:65.

11. Li W, Feng Y, Zhang L, Xiao L. Potential impacts of host specificity on zoonotic or interspecies transmission of Enterocytozoon bieneusi. Infect Genet Evol. 2019;75:104033.

12. Shen Y, Gong B, Liu X, et al. First identification and genotyping of Enterocytozoon bieneusi in humans in Myanmar. BMC Microbiol. 2020;20:10.

13. Du S, Population structure of Giardia lamblia, Cryptosporidium spp. and Enterocytozoon bieneusi in some rare animals in Qinling Mountains, China. Thesis MS. Northwest A\&F University, Xianyang City, Shannxi, China, 2015,65.

14. Feng Y, Li N, Dearen T, et al. Development of a multilocus sequence typing tool Sfor high-resolution genotyping of Enterocytozoon bieneusi. Appl Environ Microbiol. 2011;77:4822-8.

15. Liu X, Wu Y, Yang F, et al. Multilocus sequence typing of enterocytozoon bieneusi isolates from various mammal and bird species and assessment of population structure and substructure. Front Microbiol. 2020;11:1406.

16. Karim MR, Dong H, Li T, et al. Predomination and new genotypes of enterocytozoon bieneusi in captive nonhuman primates in zoos in china: High genetic diversity and zoonotic significance. PLoS One. 2015;10:e117991.

17. Karim MR, Rume FI, Rahman ANMA, et al. Evidence for zoonotic potential of enterocytozoon bieneusi in its first molecular characterization in captive mammals at bangladesh national zoo. J Eukaryot Microbiol. 2020;67:427-35.

18. Karim MR, Wang R, Dong H, et al. Genetic Polymorphism and Zoonotic Potential of Enterocytozoon bieneusi from Nonhuman Primates in China. Appl Environ Microb. 2014;80:1893-8.

19. Li W, Deng L, Yu X, et al. Multilocus genotypes and broad host-range of Enterocytozoon bieneusi in captive wildlife at zoological gardens in China. Parasite Vector. 2016;9:395.

20. Li W, Kiulia NM, Mwenda JM, et al. Cyclospora papionis, Cryptosporidium hominis, and HumanPathogenic Enterocytozoon bieneusi in Captive Baboons in Kenya. J Clin Microbiol. 2011;49:4326-9. 
21. Mynarova A, Foitova I, Kvac M, et al. Prevalence of Cryptosporidium spp., Enterocytozoon bieneusi, Encephalitozoon spp. And Giardia intestinalis in Wild, Semi-Wild and Captive Orangutans (Pongo abelii and Pongo pygmaeus) on Sumatra and Borneo, Indonesia. PLoS One. 2016;11:e152771.

22. Sak B, Kvac M, Petrzelkova K, et al. Diversity of microsporidia (Fungi: Microsporidia) among captive great apes in European zoos and African sanctuaries: Evidence for zoonotic transmission? Folia Parasit. 2011;58:81-6.

23. Sak B, Petrželková KJ, Květoňová D, et al. Diversity of microsporidia, Cryptosporidium and Giardia in mountain gorillas (Gorilla beringei beringei) in Volcanoes National Park, Rwanda. PLoS One. 2014;9:e109751.

24. Sak B, Petrzelkova KJ, Kvetonova D, et al. Long-Term monitoring of microsporidia, cryptosporidium and giardia infections in western lowland gorillas (Gorilla gorilla gorilla) at different stages of habituation in dzanga sangha protected areas, central african republic. PLoS One. 2013;8:e71840.

25. Ye J, Xiao L, Ma J, et al. Anthroponotic enteric parasites in monkeys in public park, China. Emerg Infect Dis. 2012;18:1640-3.

26. Yu F, Wu Y, Li T, et al. High prevalence of Enterocytozoon bieneusi zoonotic genotype $D$ in captive golden snub-nosed monkey (Rhinopithecus roxellanae) in zoos in China. BMC Vet Res. 2017;13:158.

27. Zhao W, Yu S, Yang Z, et al. Genotyping of Enterocytozoon bieneusi (Microsporidia) isolated from various birds in China. Infection Genetics Evolution. 2016;40:151-4.

28. Tian G, Zhao G, Du S, et al. First report of Enterocytozoon bieneusi from giant pandas (Ailuropoda melanoleuca) and red pandas (Ailurus fulgens) in China. Infection genetics evolution. 2015;34:32-5.

29. Wang L, Zhang H, Zhao X, et al. Zoonotic Cryptosporidium Species and Enterocytozoon bieneusi Genotypes in HIV-Positive Patients on Antiretroviral Therapy. J Clin Microbiol. 2012;51:557-63.

30. Buckholt MA, Lee JH, Tzipori S. Prevalence of Enterocytozoon bieneusi in swine: An 18-month survey at a slaughterhouse in Massachusetts. Appl Environ Microbiol. 2002;68:2595-9.

31. Sulaiman IM, Fayer R, Lal AA, et al. Molecular Characterization of Microsporidia Indicates that Wild Mammals Harbor Host-Adapted Enterocytozoon spp. As well as Human-Pathogenic Enterocytozoon bieneusi. Appl Environ Microb. 2003;69:4495-501.

32. SANTÍN M. FAYER R. Enterocytozoon bieneusi Genotype Nomenclature Based on the Internal Transcribed Spacer Sequence: A Consensus. J Eukaryot Microbiol. 2009;56:34-8.

33. Yu Z, Wen X, Huang X, et al. Molecular characterization and zoonotic potential of Enterocytozoon bieneusi, Giardia duodenalis and Cryptosporidium sp. In farmed masked palm civets (Paguma larvata) in southern China. Parasite Vector. 2020;13:1-10.

34. Xu C, Ma X, Zhang H, et al. Prevalence, risk factors and molecular characterization of Enterocytozoon bieneusi in raccoon dogs (Nyctereutes procyonoides) in five provinces of Northern China. Acta Trop. 2016;161:68-72.

35. Yang Y, Lin Y, Li Q, et al. Widespread presence of human-pathogenic Enterocytozoon bieneusi genotype $D$ in farmed foxes (Vulpes vulpes) and raccoon dogs (Nyctereutes procyonoides) in China: First identification and zoonotic concern. Parasitol Res. 2015;114:4341-8. 
36. Zhang X, Cong W, Liu G, et al. Prevalence and genotypes of Enterocytozoon bieneusi in sika deer in Jilin province, Northeastern China. Acta Parasitol. 2016;61:382-8.

37. Su B, Fu Y, Wang Y, Jin L, Chakraborty R. Genetic Diversity and Population History of the Red Panda (Ailurus fulgens) as Inferred from Mitochondrial DNA Sequence Variations. Mol Biol Evol. 2001;18:1070-6.

38. Zhong Z, Tian Y, Song Y, et al. Molecular characterization and multi-locus genotypes of Enterocytozoon bieneusi from captive red kangaroos (Macropus Rfus) in Jiangsu province, China. PLoS One. 2017;12:e183249.

39. Li W, Xiao L. Ecological and public health significance of Enterocytozoon bieneusi. One Health. 2021;12:100209.

40. Wang H, Liu Q, Jiang X, et al. Dominance of zoonotic genotype D of Enterocytozoon bieneusi in bamboo rats (Rhizomys sinensis). Infection genetics evolution. 2019;73:113-8.

41. Deng L, Li W, Yu X, et al. First Report of the Human-Pathogenic Enterocytozoon bieneusi from RedBellied Tree Squirrels (Callosciurus erythraeus) in Sichuan, China. PLoS One. 2016;11:e163605.

42. Deng L, Li W, Zhong Z, et al. Multi-locus genotypes of Enterocytozoon bieneusi in captive Asiatic black bears in southwestern China: High genetic diversity, broad host range, and zoonotic potential. PLoS One. 2017;12:e171772.

43. Li W, Xiao L. Multilocus Sequence Typing and Population Genetic Analysis of Enterocytozoon bieneusi: Host Specificity and its Impacts on Public Health. Front Genet. 2019;10:307.

44. Deng L, Li W, Zhong Z, et al. Molecular characterization and multilocus genotypes of Enterocytozoon bieneusi among horses in southwestern China. Parasit Vectors. 2016;9:561.

45. Karim MR, Wang R, He X, et al. Multilocus sequence typing of Enterocytozoon bieneusi in nonhuman primates in China. Vet Parasitol. 2014;200:13-23.

\section{Figures}




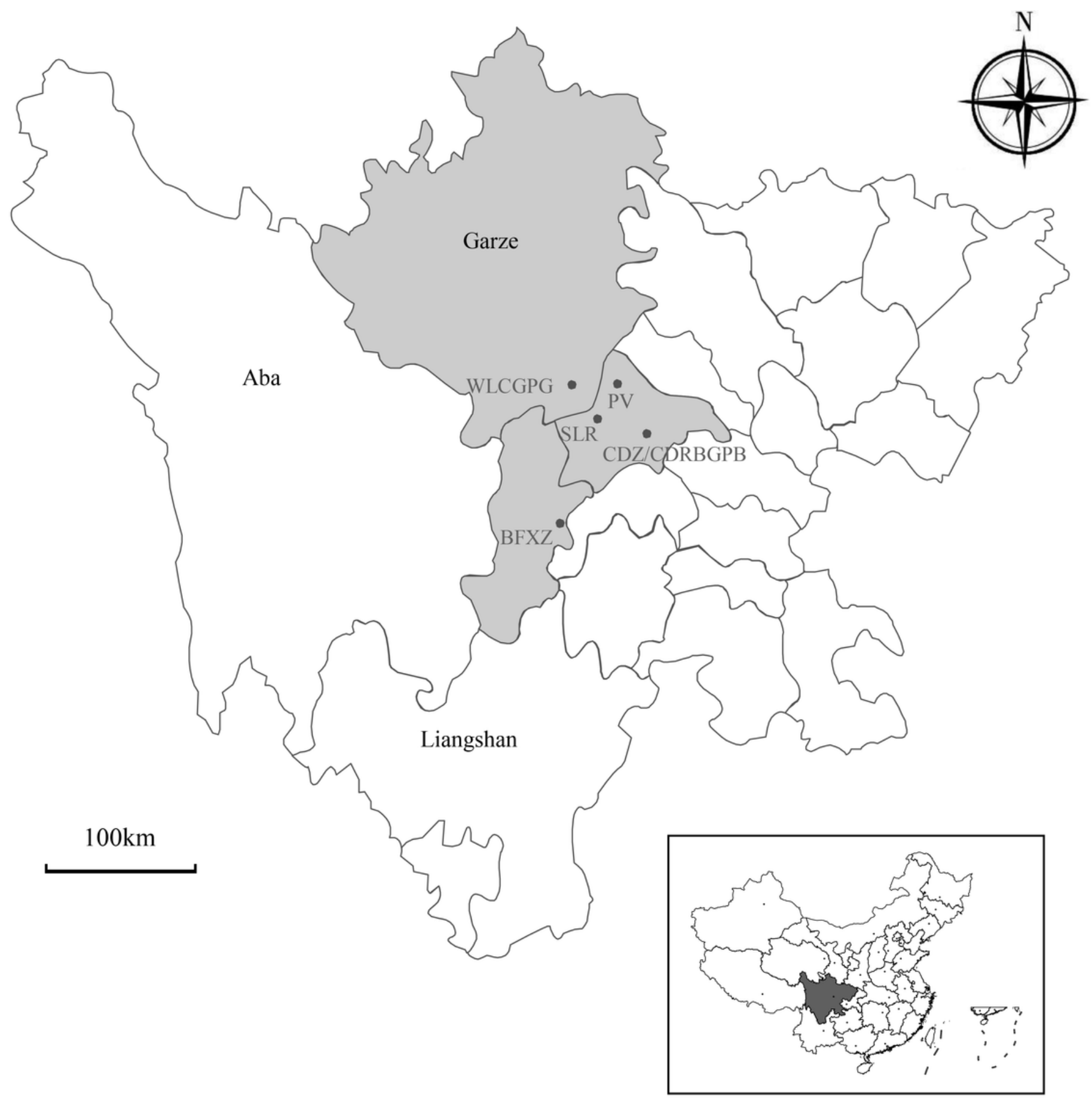

Figure 1

The sampling sites of captive red pandas in Sichuan province, southwest China. 


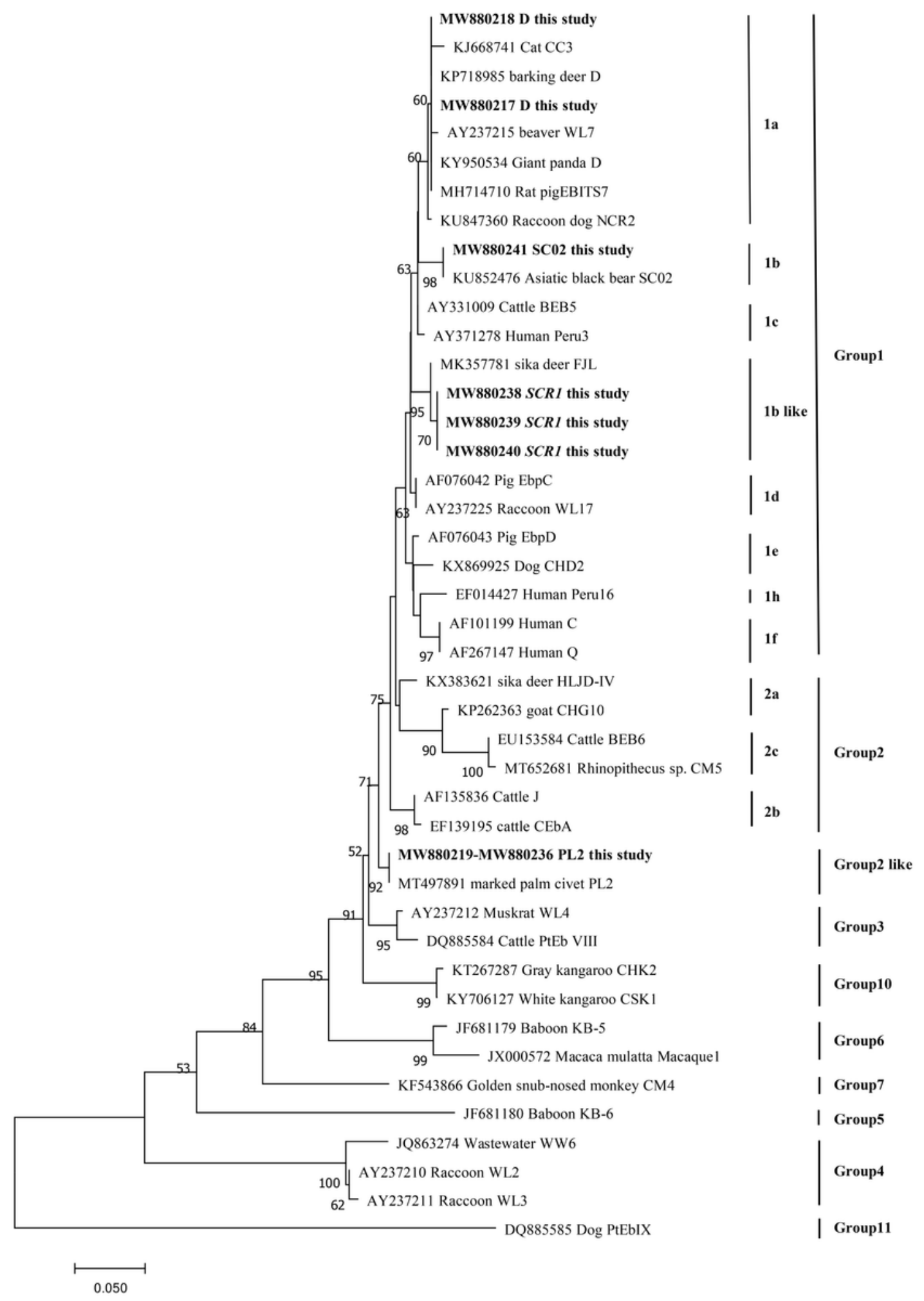

\section{Figure 2}

Phylogenetic tree of Enterocytozoon bieneusi genotypes based on ITS sequences analyzing. Phylogenetic relationships between obtained sequences in the present study and other sequences downloaded in GenBank. The known and novel E. bieneusi genotypes identified in this study are shown in bold and the novel genotypes are shown in italic. Genotype PtEb IX (DQ885585) from dogs was used as an outgroup. 